\title{
Application Design of Electrical Fire Monitoring System Based on ZigBee Wireless Technology
}

\author{
ZHANG ZunYang ${ }^{1, a}$, DING LiMing ${ }^{2, b}$ \\ 1. 2 North Minzu University, Yinchuan,Ningxia,750021 China \\ a,b522122145@qq.com
}

Keywords: grading warning; fire probability; ZigBee; multi-sensor fusion

\begin{abstract}
In view of the present situation where traditional fire alarm system are insufficient in the wiring and the capacity and wireless fire warning products are in short, a wireless fire probability grading warning system is designed using wireless sensor network technology and multi-sensor fusion technology; this system uses ZigBee technology to establish star forecasting and warning network, and provides warning to the fire catastrophic tendency according to the probability based on multi-sensor fusion information, so as to adapt to the fire early warning and alarm in various complex environments.
\end{abstract}

\section{Introduction}

Among various kinds of disasters, fire is one of the main disasters which is the most frequent, and the most common threat to public safety; its harm and loss is becoming more and more serious, and therefore, the development of fire prevention and monitoring technology is an inevitable trend. As for fire prevention, our country mostly judge according to the analysis on fire risk and provides classified warning from such aspects as material flammability, storage and using method, etc, which causes the lack in the classification consciousness and the experience in fire hazards and wins valuable time for eliminating fire in the bud.

\section{ZigBee network topology}

In ZigBee protocol stack, 3 kinds of topological forms are defined as Figure 1, which includes star topology, cluster topology and reticular topology.
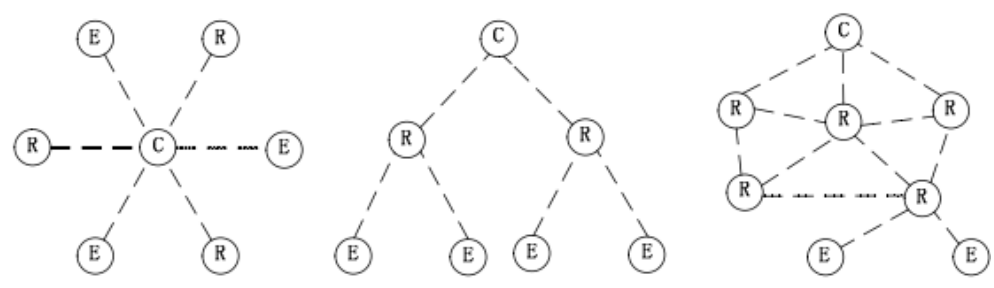

(a) Star topology (b) Cluster topology (c) Reticular topology

Figure 1 ZigBee Network Topological Structure

Star topology is the simplest in the three kinds of topology structure; this kind of structure contains a master device and multiple branch devices (ZigBee routing nodes or ZigBee terminal nodes); the function of master device is to establish and maintain network. In this kind of network, the master device can communicate with any one of the branch device; direct communication cannot be realized among branch device, and the communication with other branch device can only be through master device, and therefore, this kind of network structure is called as single-hop network. At the time of establishing network, ZigBee coordinator will initialize some parameters to determine the structure type of the network. Just as star topology, cluster topology and reticular topology also contain master device and branch devices (routing nodes and terminal nodes). in cluster topoly, the communication mainly develops with grading strategies relying on routing device, sending data now and then; if there are mal-functions in routing nodes, the corresponding 
terminal nodes will build network from the beginning. The routing function of reticular topology is relatively flexible and routing devices can communicate with each other directly, to realize the recticular function of routing and to increase the stability of network. In the network layer, a constant nwkc Coordinator Capable is provided to indicate whether the device has the capability of coordinator; 0x00 indicates that the device cannot be taken as coordinator. ZigBee technology is a kind of close-range wireless communication technology with low power consumption and low speed. At the time of arranging fireproof monitoring system inside the building, it is necessary to consider such aspects as the communication range, power consumption, energy saving device of the system; using ZigBee technology includes these advantages: (1) network flexibility, good extension performance.(2) low power consumption and easy maintenance.

Table 1 Features of multiple wireless communication techniques

\begin{tabular}{|c|l|}
\hline $\begin{array}{c}\text { Wireless } \\
\text { communication } \\
\text { technique }\end{array}$ & \multicolumn{1}{c|}{ Application characteristics } \\
\hline $\begin{array}{c}\text { Infrared } \\
\text { technology }\end{array}$ & $\begin{array}{l}\text { Opaque object will disturb the communication among nodes and it is usually } \\
\text { used in point-to-point transmission communication. }\end{array}$ \\
\hline Wifi & $\begin{array}{l}\text { The network capacity is small, and it is generally used in short distance mobile } \\
\text { device communication. }\end{array}$ \\
\hline Bluetooth & $\begin{array}{l}\text { It is generally used in wireless short distance LAN, there is a high transmission } \\
\text { rate. }\end{array}$ \\
\hline ZigBee & $\begin{array}{l}\text { Energy dissipation, speed and cost are low enough to be widely used in } \\
\text { wireless domain networks. }\end{array}$ \\
\hline
\end{tabular}

(3)Strong fault tolerance. ZigBee, as a wireless communication technology, can make further analysis on the monitoring data by using information fusion, which can improve the accuracy of the data, and ZigBee wireless communication technology is chosen.

\section{Design and implementation of electrical fire monitoring system monitor}

In the electrical fire monitoring system, the monitor device is responsible for the conversion and judgment of the information collected by the residual current detector and temperature detector. If the measured value exceeds the preset value, the sound light alarm will be carried out, and the failure warning information will be stored.

A. Brief introduction to the MSP430 series Single Chip Microcomputer. The MSP430 series is a 16-bit hybrid Single Chip Microcomputer with low power consumption and a streamlined instruction set. The rapid development and application scope of MSP430 series Single Chip Microcomputer is mainly based on the following characteristics: (1) powerful processing power;(2) quick operation;(3) abundant interruption resources;(4) low power consumption;(5) rich peripheral modules; (6) convenient and efficient development environment.

B. Collection of residual current. Current transformer converts residual current signal into voltage signal. Due to alternating signals come out of the current transformer and the waveform is not smooth, rectification is necessary for alternating signals to pass through precision rectifier rectifier circuit before entering the MCU and $\mathrm{AD}$; alternating signals can conduct filtering through adding capacitance at the MCU AD pins add after rectification.The precision rectifier circuit is shown in figure 2. 


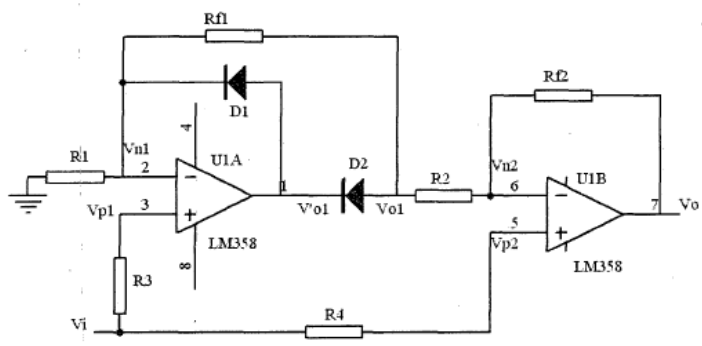

Figure 2 Precision rectification circuit

The relationship between the input voltage and output voltage is shown as:

$$
V o= \begin{cases}+V i & \text { 当 } V i>0 \\ -V i & \text { 当 } V i<0\end{cases}
$$

In Figure 1, at the in-phase input terminal, UlA composes the in-phase amplifier, and the U1B compose the differential amplifier. Input voltage is added to the operational amplifier with high input resistance. When the input voltage is >> 0, D1 guides, and D2 cuts off; then UlA composes the voltage follower, which is the anti-phase terminal of the Rf 1 and R2 to the UlB; therefore, the output voltage of UlB is:

$$
V o=\left(1+\frac{R f 2}{R f 1+R 2}\right) V i-\frac{R f 2}{R f 1+R 2} V i=V i
$$

When the input voltage is $<0$, D1 closes, D2 leads, and then UlA is the same phase amplifier.

$$
V o l=\left(1+\frac{R f 1}{R 1}\right) V i
$$

The output voltage of U1B is:

$$
V o=\left(1+\frac{R f 2}{R f 1+R 2}\right) V i-\frac{R f 2}{R f 1+R 2} V i=V i
$$

The voltage transmission characteristics of the circuit are shown in figure 3.

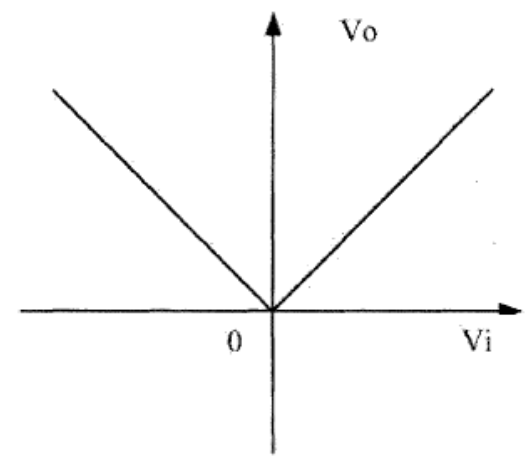

Figure 3: Precision full-wave rectified voltage transmission characteristics

The voltage value gained from the zero sequence current transformer and the residual current value of the actual electrical wiring forms linear relationship; therefore, multiply the voltage value gained from the zero sequence current transformer read from MCU and $\mathrm{AD}$ with certain fixed coefficient, we can get the residual current value. Of course, this coefficient varies with the zero sequence current transformer; therefore, this value should be determined before measurement and be stored in related storage; in this way, each time when MCU starts, the real residual current value can be calculated according to this value.

C. Temperature measurement. There are many options for temperature detectors, which are generally divided into two basic modes: thermocouple and thermal resistance. If you choose a thermocouple, you need to add an integrated module such as MAX6675, which is not helpful for reducing the cost of the product. In this design, with so many factors, platinum resistors are used to 
measure temperature. Platinum resistance temperature sensor is produced according to the functional relation between the resistance and the temperature; due to its high accuracy, large measuring range, good reproducibility and stability, etc., it is widely used in the temperature measurement of medium temperature $(-2000$ - C - 2000 - C) range.The curve of temperature-resistor of PT100 is shown in Figure 4.

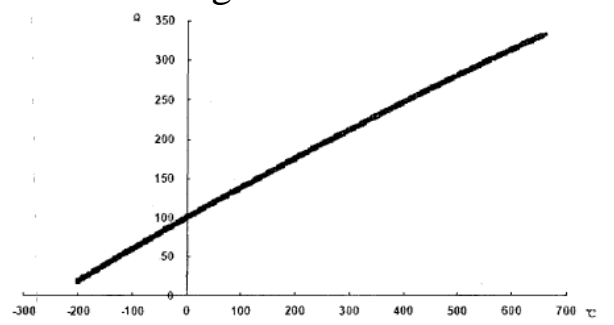

Figure 4 The curve of temperature-resistor of PT100

In the figure above, we can see that the relation between the resistance value of PT100 and the temperature is linear. This kind of non-strict linear property between the resistance and the temperature of the platinum resistance will lead to a certain error in the measurement of temperature. Hardware circuits are often used to reduce this error during early period. The temperature measurement system circuit is shown in Figure 4. The circuit adopts an unbalanced bridge to measure the voltage signal of platinum resistance with temperature change. After amplification and A/D conversion, the circuit is sent to the MCU for processing. The RT represents PT100.

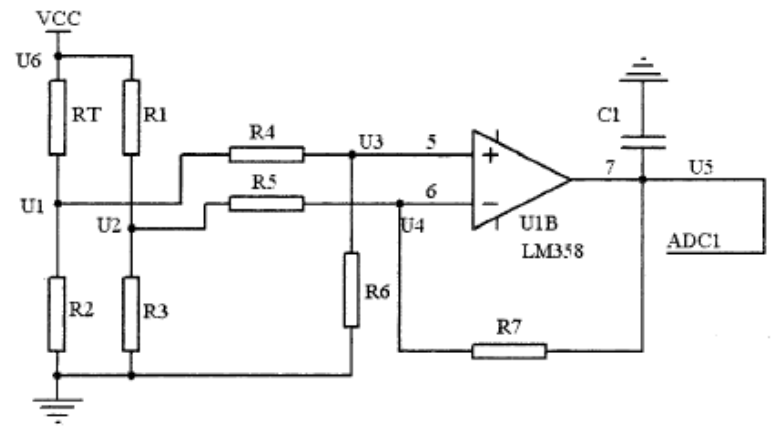

Figure 5 Temperature measuring circuit

D. Display circuit design. In the electrical fire monitoring system, it is necessary to use display equipment to browse the status information, and to conduct the setting and reading of parameters of the monitor equipment. In addition, when there is an alarm or a fault signals, a display screen is required to display the information. In this design, LCD screen is adopted to realize the key. The hardware circuit is shown in figure 6.

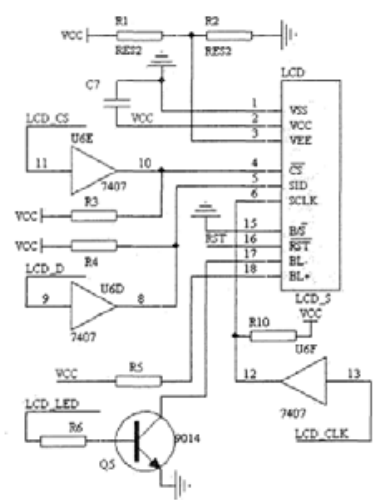

Figure 6 Display circuit

In Figure 6, LCD adopts $12232 \mathrm{~F} ; 12232 \mathrm{E}$ is a LCD with a built-in 8192 points of $16 * 16$ Chinese character and 128 points of $16 * 8$ ASCII character set graphic dot matrix; it is mainly composed of row driver/ column driver and $128 * 32$ whole dot matrix LCD display. It can display graphs and also $7.5 * 2$ characters. The interface with outer CPU uses parallel or serial method to control. In this design, 12232E uses serial way to communicate with the single-chip microcomputer, 
i.e. a single clock line and a data line are used for data exchange. In the Figure, R72 and R73 are for contrast adjustment. The reading and the writing time slot $12232 \mathrm{E}$ serial port are shown in Figure 7 and Figure 8.

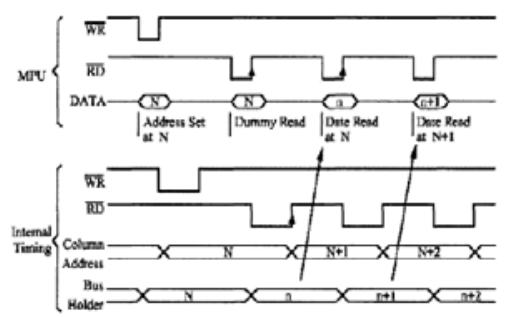

Figure 7 Reading time slot of 12232E serial port

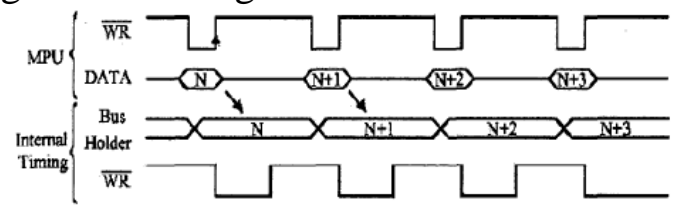

Figure 8 Writing time slot of 12232E serial port

\section{Conclusions}

Through the combination of the ZigBee technology and the multi-sensor fusion technology, this paper designs a warning system for real-time monitoring on fire, which can makes classified warning on fire at the preliminary stage; this design scheme has been applied to practice, but the stability, robustness and delay of system need to be enhanced; new improvements will be made on the algorithm and processor in the late stage; we hope to initiate new direction for the fire prevention.

\section{References}

[1] Zhou Keliang, Guo Jinmei, Nie Lei. Design and Realization of Electrical Wireless Fire Infrared Measurement System Based on STM32F [J]. Computer Measurement and Control, 2014,10:3108-3110.

[2] Yang Fan. Design and Realization of Monitoring System Based on ZIGBEE Wireless Network Technology. [J]. Scientific and Technical Information, 2013,35:49-52.

[3] Wu Longliang, Xue Nan, Quan Yikui, Chao Lumen, Liu Wanyu, Yang Bing. Design of Fire Warning System Based on ZigBee and LabVIEW[J]. Microcomputer and Application, 2013,22:53-55.

[4] Wang Liuliu, Liao Xiaowei, Shen Xiaobo. Design of Grading Warning System of Wireless Fire Probability Based on ZigBee Technique [J]. Chongqing Technology and Business University Journal (Natural Science) ,2013,07:57-61.

[5] Chu Yanwei, Yang Bo, Liu Heng. Intelligent Wireless Temperature Meaurement System of High-voltage Switch Cabinet Based on ZigBee [J]. Electrical \& Electronics,2013,06:23-28. 